\title{
Solving Airline Operations Problems Using Specialized Agents in a Distributed Multi-Agent System
}

\author{
António J.M. Castro and Eugénio Oliveira \\ LIACC-NIADR\&R, Faculty of Engineering, University of Porto \\ R. Dr. Roberto Frias, 4200-465 Porto, Portugal \\ $\{a j m c, e c o\} @$ fe.up.pt
}

\begin{abstract}
An airline schedule very rarely operates as planned. Problems related with aircrafts, crew members and passengers are common and the actions towards the solution of these problems are usually known as operations recovery. The Airline Operations Control Center (AOCC) tries to solve these problems with the minimum cost and satisfying all the required rules. In this paper we present the implementation of a Distributed Multi-Agent System (MAS) representing the existing roles in an AOCC. This MAS deals with several operational bases and for each type of operation problems it has several specialized software agents that implement different algorithms (heuristic, AI, $\mathrm{OR}$, etc.), competing to find the best solution for each problem. We present a real case study taken from an AOCC where a crew recovery problem is solved. Computational results using a real airline schedule are presented, including a comparison with a solution for the same problem found by the human operators in the AOCC. We show that, even in simple problems and when comparing with solutions found by human operators, it is possible to find valid solutions, in less time and with a smaller cost.
\end{abstract}

Keywords: Distributed Multi-Agent Systems, Airline Operations Control, Operations Recovery, Disruption Management.

\section{Introduction}

One of the most important concerns in an airline company is the Operations Control. Through operations control mechanisms the airline company monitors all the flights checking if they follow the schedule that was previously defined by other areas of the company. Unfortunately, some problems arise during this phase [8]. Those problems are related with crew members (for example, a crew member that did not report for duty), aircrafts (for example, a malfunction or a delay due to bad weather) and passengers. When any of these problems appear it is necessary to find solutions for them. The Airline Operations Control Centre (AOCC) is composed by teams of people specialized in solving the above problems under the supervision of an operation control manager. Each team has a specific goal (for example, to guarantee that each flight has the necessary crew members) contributing to the common and general goal of having the airline operation running with few problems as possible. The process of solving these problems is known as Disruption Management [7] or Operations Recovery.

J. Filipe, J. Cordeiro, and J. Cardoso (Eds.): ICEIS 2007, LNBIP 12, pp. 173-184, 2008.

(C) Springer-Verlag Berlin Heidelberg 2008 
Based on the observations we have done on an AOCC of a real airline company we hypothesize that the objective of solving the operations recovery problems with the less cost as possible, will be much easier to achieve if we include information in the decision process related with various costs as well as if we take advantage of the fact that airlines usually have different operational bases with specific resources. Regarding crew recovery problems, we predict that if we take into account payroll information like hour salary and perdiem value of each crew rank, and costs related with hotels and extra-crew travel between the different operational bases, the solution will be less expensive. The same principle can be applied to aircraft recovery and passenger recovery if we use costs related with that domain. We also hypothesize that the use of different algorithms to solve the same problem (in crew and aircraft recovery) will contribute to the robustness of the system. We predict that using different algorithms (genetic algorithms, heuristic, etc.) in comparison with using always the same algorithm, to solve the same problem, will permit to always find the best solution (according to the criteria defined by the company) and to always find $\boldsymbol{a}$ solution, especially taking into account the fact that we might benefit from solutions presented by other operational bases.

In this paper we approach this problem so that it can be solved by a Multi-agent System (MAS) that represents the Operational Control Center of the airline company. We use specialized agents, each one implementing Artificial Intelligence algorithms, simple heuristic algorithms and/or Operations Research mathematical models, to find the best solution to a specific problem related with crew, aircrafts or passengers. We expect to obtain a considerable decrease in the costs of the solutions for the problems found when compared with the costs of the solutions found by the current method used in the airline we have observed. We also expect that the heterogeneity of the algorithms, specialized in different types of problems, will allow to find solutions especially for the non-trivial problems, contributing, in this way, to the robustness of the system.

The rest of the paper is structure in the following way. Section 2 presents some work of other authors regarding operations recovery. Section 3 presents our proposal of a MAS for airline operations recovery, including the architecture of the MAS, the algorithm used to choose the best solution and an example of the application of our MAS. Section 4 presents the scenario we setup to evaluate our system as well as the results of the evaluation. Section 5 presents the conclusion of our work.

\section{Related Work}

Traditionally, the Operations Recovery Problem has been solved through Operations Research (OR) techniques. The paper [2] gives on overview of OR applications in the air transport industry. The literature that exists related with this subject is usually divided according to the specific resource to be recovered. The most common division is by aircraft, crew and passengers. However, it is also possible to find papers related with more general approaches as well as related with integrated recovery approaches. We will present here the most recent published papers according to [6]. We divided the papers in four areas: general approaches, aircraft recovery, crew recovery and integrated recovery. For a more detailed explanation of the papers as well as for older papers related with each of these subjects, please consult [6]. 
General Approaches: In [7] the author reports on the experiences obtained during the research and development of project DESCARTES (a large scale project supported by EU) on airline disruption management. The current (almost manual) mode of dealing with recovery is presented. They also present the results of the first prototype of a multiple resource decision support system.

Aircraft Recovery: The most recent paper considering the case of aircraft recovery is dated from 2002 [12]. The proposed model addresses each aircraft type as a single problem. They formulate the problem as a Set Partitioning master problem and a route generating procedure. The goal is to minimize the cost of cancellation and retiming, and it is the responsibility of the controllers to define the parameters accordingly. It is included in the paper a testing using SimAir [13] simulating 500 days of operations for three fleets ranging in size from 32 to 96 aircraft servicing 139-407 flights.

Crew Recovery: In [1] the flight crew recovery problem for an airline with a hub-andspoke (a system of air transportation in which local airports offers air transportation to a central airport where long-distance flights are available) network structure is addressed. The paper details and sub-divides the recovery problem into four categories: misplacement problems, rest problems, duty problems, and unassigned problems. Several means are used for recovery, including delaying, swapping, deadheading (extra-crew) and the use of stand by crew. Results are presented for a situation from a US airline with 18 problems.

Integrated Recovery: It is uncommon to find literature dedicated specifically to the passenger recovery problem. We believe the main reason for this is the fact that the passenger problems can be minimized if we solve the aircraft and crew problems. However, we would like to point out a recent paper [4] that, although presenting an integrated recovery approach, has a strong emphasis on reducing passenger arrival delays. This paper presents two models that considers aircraft and crew recovery and through the objective function focuses on passenger recovery. To test the models an AOCC simulator was developed, simulating domestic operations of a major US airline. It involves 302 aircrafts divided into 4 fleets, 74 airports and 3 hubs. Furthermore, 83869 passengers on 9925 different passengers' itineraries per day are used. Three different scenarios with different levels of disruption are presented. For all scenarios are generated solutions with reductions in passenger delays and disruptions.

Lettovsky's Ph.D. thesis [9] is the first presentation of a truly integrated approach in the literature, although only parts of it are implemented. The thesis presents a linear mixed-integer mathematical problem that maximizes total profit to the airline while capturing availability of the three most important resources: aircraft, crew and passengers. The formulation has three parts corresponding to each of the resources, that is, crew assignment, aircraft routing and passenger flow. In a decomposition scheme these three parts are controlled by a master problem denominated the Schedule Recovery Model.

Finally, we would like to point out a tool called DART (Decision-Aided Rescheduling Tool) [11] that was developed to control the flight operations of IBERIA (the Spanish airline company). DART controls airline operations by gathering real time world-wide information about fleet and crew situation and providing decision support for handling incidents. It covers the daily execution of the ideal flight plan and is 
responsible for tracking and solving any irregularities that might arise during its execution. The authors claim that DART has been able to solve some difficult problems, proposing, in some cases, better solutions than those proposed by the rescheduling experts. The paper does not present any comparative results.

In addition to the above literature the conferences of the AGIFORS ${ }^{1}$ organization often feature presentations related with operations recovery. The contributions from these conferences are, at best, available in the form of presentation slides. As such, we did not consider them here.

\section{Airline Operations Recovery through an Multi-Agent System}

\subsection{General Description}

As stated before we approached this problem by developing a distributed MAS that represents the AOCC. A high-level graphical representation of the MAS architecture is presented in Fig. 1.

The square labeled BASE A shows the part of the MAS that is installed in each operational base of the airline company (e.g., NYC, LHR and LAX). Each operational base has its own resources that are represented in the environment, for example, Crew Roster and Aircraft Roster are databases of schedules for the crew members and aircrafts, respectively. Other resources represented are the airport information system

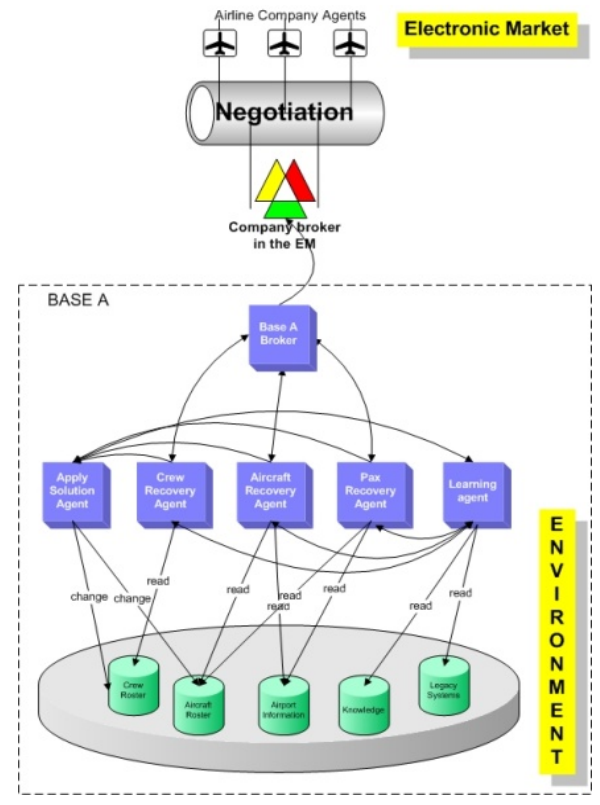

Fig. 1. MAS architecture

\footnotetext{
${ }^{1}$ AGIFORS, http://www.agifors.org, November 2007.
} 
(to be able to get information regarding boarding gates and delays), legacy systems (to access information regarding costs, among others) and a knowledge database for the learning capabilities of the MAS (this characteristic of the MAS will not be explained in this paper). Each operational base has also software agents that represent roles in the AOCC. The Crew Recovery Agent, Aircraft Recovery Agent and Pax Recovery Agent are dedicated to solve crew, aircraft and passengers problems, respectively, and should be seen as sub-organizations inside the MAS. The Apply Solution Agent applies the solution found and authorized in the resources of the operational base. The Learning Agent is dedicated to the learning capabilities of the MAS. The MAS has also the possibility to interact with an electronic market of airline resources such as aircrafts and crew members, through the Company Broker. According to [7] "research on recovery operation to this date only deals with a single airline. Cooperation between airlines is not supported". With this approach we try to foster the cooperation between airlines. More information about this electronic market can be found in [10].

The MAS was developed using JADE [3] as development platform and as the runtime environment that provides the basic services for agents to execute. The MAS was developed based on a previous analysis and design by Castro and Oliveira [5].

\subsection{Sub-organization Architecture}

As stated before, the Crew, Aircraft and Pax Recovery agents as presented in Fig. 1 should be seen as sub-organizations. These sub-organizations have their own architecture with their specialized agents. Fig. 2 shows the architecture for Crew Recovery in a UML diagram. The architecture for Aircraft Recovery and Pax Recovery are very similar.

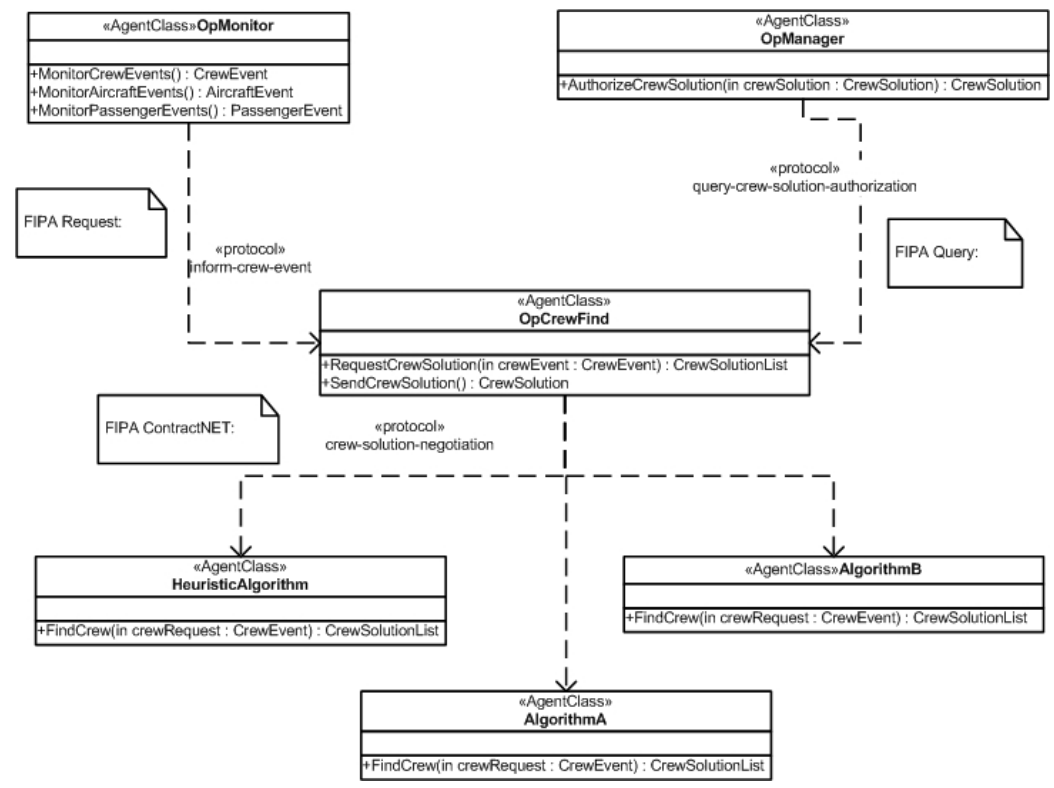

Fig. 2. Crew Recovery sub-organization architecture 


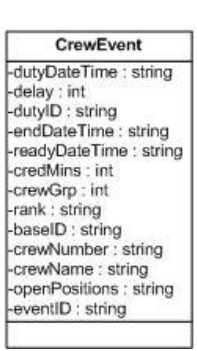

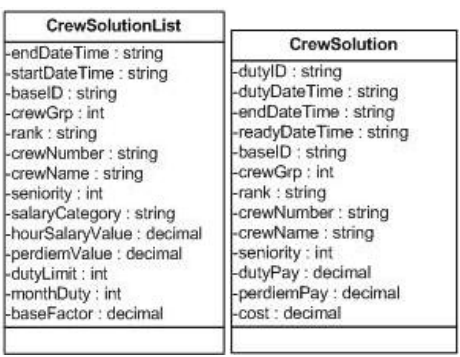

Fig. 3. Crew Events

The agent class OpMonitor is responsible for monitoring any crew events, for example, crew members that did not report for duty or duties with open positions, that is, without any crew member assigned to a specific role on board (e.g., captain or flight attendant). When an event is detected, the service MonitorCrewEvents will initiate the protocol inform-crew-event (FIPA Request) informing the OpCrewFind agent. The message will include the information necessary to characterize the event. This information is passed as a serializable object of the type CrewEvent. Fig. 3 shows the attributes of the CrewEvent class.

The OpCrewFind agent detects the message and will start a CFP (call for proposal) through the crew-solution-negotiation protocol (FIPA contractNET) requesting to the specialized agents HeuristicAlgorithm, AlgorithmA and AlgorithmB of any operational base of the airline company, a list of solutions for the problem. Each agent implements a different algorithm specific for this type of problem. When a solution is found a serializable object of the type CrewSolutionList is returned in the message as an answer to the CFP. Fig. 3 shows the attributes of the CrewSolutionList class.

The OpCrewFind agent collects all the proposals received and chooses the best one according to the algorithm in Table 1:

Table 1. Multi-criteria algorithm

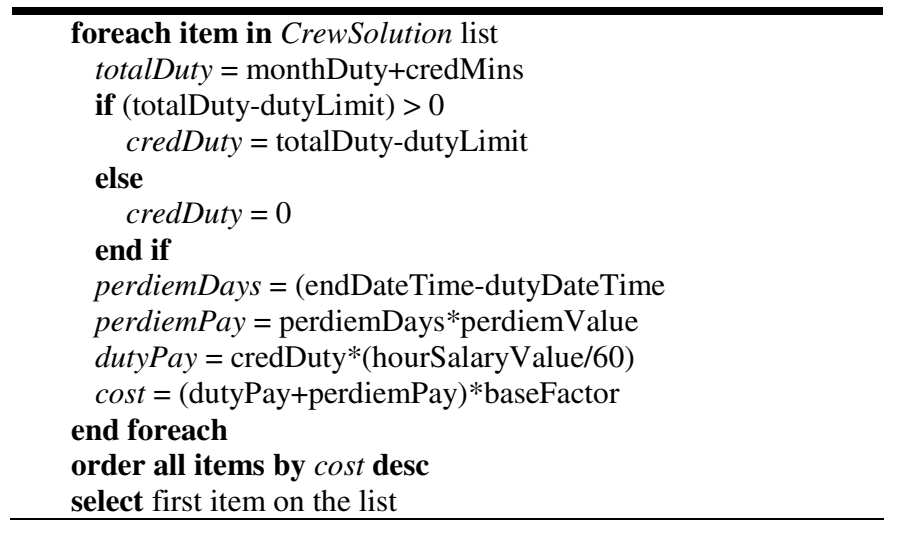


Table 2. Computed values

\begin{tabular}{|l|l|}
\hline totalDuty & Monthly duty minutes of the proposed crew after assigning the new duty \\
\hline credDuty & Number of minutes to be paid case the crew exceeds the monthly duty limit \\
\hline dutyPay & Cost of duty computed according to the hour salary of the crew \\
\hline perdiemDays & Number of days of work for the specific duty \\
\hline perdiemPay & Cost of duty computed according to the perdiem value of the crew \\
\hline baseFactor & The cost associated with the base (extra-crew, hotels, etc.) \\
\hline Cost & The sum of the cost of the perdiem plus duty multiplied by the base factor. \\
\hline
\end{tabular}

The algorithm in Table 1 is implemented in the service SendCrewSolution and produces a list ordered by the cost (a multi-criteria cost) that each solution represents. Table 2 explains each of the computed values in the algorithm in Table 1.

The first solution of the list in descendant order by cost corresponds to the less expensive one. The SendCrewSolution service initiates the protocol query-crewsolution-authorization (FIPA Query) querying the OpManager agent for authorization. The message includes the serializable object of the type CrewSolution as shown in Fig. 3.

\subsection{Example}

Consider the following situation: Airline Company A has two operational bases, one in London (LHR) and another in Paris (ORY), each with 150 crew members. On a specific day a crew member of the LHR base did not report for duty and it was necessary to find another crew member. In our MAS the OpMonitor agent of LHR base, would detect and characterize the event according to Table 3 .

The agent starts the inform-crew-event protocol that includes the information from the CrewEvent, informing the OpCrewFind agent. This agent starts a CFP through the crew-solution-negotiation protocol requesting all the solutions from the specialized agents in both operational bases. The OpCrewFind agent receives the solutions as a CrewSolutionList from each agent according to Table 4 (this table does not show all the information that is included in the CrewSolutionList returned by the agents, like for example, the crew number and name).

The service SendCrewSolution of agent OpCrewFind computes the values indicated in Table 2 for each item of the CrewSolutionList and orders them, according to the algorithm indicated in Table 1 . The result is indicated in Table 5.

Table 3. Event characterization

\begin{tabular}{llllll}
\hline Attribute & Value & Comment & Attribute & Value & Comment \\
\hline dutyDateTime & $05-10-2006$ 10:00 & & rank & FA & Crew rank \\
\hline delay & 10 & Crew delayed 10 mins. & baseID & LHR & \\
\hline dutyID & NBPNC-1LHR19 & & crewNumber & 97 & \\
\hline endDateTime & $05-10-2006$ 20:15 & Duty end date/time & crewName & John & \\
\hline readyDateTime & $06-10-2006$ 09:15 & Includes rest & openPositions & 1 & \\
\hline credMins & 615 & Total work time. & eventID & 1230 & Internal \\
\hline crewGrp & 2 & 1=pilots; 2=flight att. & & & \\
\hline
\end{tabular}


Table 4. CrewSolutionList data

\begin{tabular}{|c|l|l|c|c|c|c|c|}
\hline$\#$ & BaseID & Rank & Hr Salary & Perdiem & Duty Limit & Month Duty & Base Factor \\
\hline 1 & LHR & FA & 43 & 71 & 7800 & 7600 & 1 \\
\hline 2 & ORY & FA & 30 & 71 & 7800 & 8120 & 1,3 \\
\hline 3 & LHR & FA & 17 & 31 & 7800 & 8500 & 1 \\
\hline 4 & LHR & FA & 14 & 31 & 7800 & 7950 & 1 \\
\hline 5 & ORY & FA & 14 & 31 & 7800 & 5000 & 1,3 \\
\hline
\end{tabular}

Table 5. Ordered CrewSolutionList data

\begin{tabular}{|c|l|c|c|c|c|c|c|}
\hline$\#$ & BaseID & Total Duty & Crd Duty & Duty Pay & Perdiem Days & Perdiem Pay & Cost \\
\hline 5 & ORY & 5615 & 0 & 0 & 1 & 31 & 40 \\
\hline 4 & LHR & 8565 & 765 & 178 & 1 & 31 & 209 \\
\hline 1 & LHR & 8215 & 415 & 297 & 1 & 71 & 368 \\
\hline 3 & LHR & 9115 & 1315 & 372 & 1 & 31 & 403 \\
\hline 2 & ORY & 8735 & 935 & 467 & 1 & 71 & 699 \\
\hline
\end{tabular}

Table 6. CrewSolution serializable object

\begin{tabular}{|l|c|c|c|}
\hline Attribute & Value & Attribute & Value \\
\hline dutyID & NBPNC-1LHR19 & crewNumber & 147 \\
\hline dutyDateTime & $05-10-200610: 00$ & crewName & Marie \\
\hline endDateTime & $05-10-200620: 15$ & seniority & 15 \\
\hline readyDateTime & $06-10-200609: 15$ & dutyPay & 0 \\
\hline baseID & ORY & perdiemPay & 31 \\
\hline crewGrp & 2 & cost & 40 \\
\hline Rank & FA & & \\
\hline
\end{tabular}

As it is possible to see, the solution with less cost is solution number 5. In this particular example, it is a crew member from a different operational base that is considered the best solution to substitute the one that did not report for duty. The SendCrewSolution service initiates the protocol query-crew-solution-authorization querying the OpManager for authorization. The message includes the serializable object CrewSolution with the complete information, as presented in Table 6.

\section{Scenario and Experiments}

\subsection{Scenario}

To evaluate our MAS we have setup a scenario that includes 3 operational bases (A, $\mathrm{B}$ and $\mathrm{C}$ ). Each base includes their crew members each one with a specific roster. The data used corresponds to the real operation of June 2006 of base A. We have simulated a situation where 15 crew members, with different ranks, did not report for duty in base A. The events did not happen at the same day and each one corresponds to a crew member that did not report for a specific duty in a specific day.

After setting-up the scenario we found the solutions for each crew event using two methods. In the first method we used a real user from the AOCC, with the current tools available, to find the solutions. The user uses software that shows the roster of 
each crew member in a Gantt chart for a specific period. The user can scroll down the information, filter according to the crew rank and base, and sort the information by name, month duty, etc. In the second method we have used the sub-organization Crew recovery of our MAS as indicated in Section 3.2.

\subsection{Results}

The data (partial) obtained using method 1 (user with current tools) is presented in Table 7 and the data obtained using method 2 in Table 8. We point out that the data in columns marked with an asterisk were calculated manually, according to the formulas in the algorithm presented in Table 1. The reason for this is that the information system that is available for the users does not include information related with any kind of payroll.

Table 9 presents the results that compare the two methods. From the results obtained we can see that in average, the second method took 25 seconds to find a solution and the first method took 101 seconds. Regarding the costs, the second method has a total cost of 3839.36 and the first method 7039.60. The second method

Table 7. Solutions obtained through method 1 (first 13 records only)

\begin{tabular}{|c|c|c|c|c|c|c|}
\hline \# & Base ID & Time (sec) & Rank & Duty Pay (*) & Perdiem Pay (*) & $\operatorname{Cos} t(*)$ \\
\hline 1 & $\mathrm{~A}$ & 90 & $\mathrm{CAB}$ & 0 & 72 & 72 \\
\hline 2 & B & 115 & $\mathrm{CAB}$ & 0 & 72 & 86,4 \\
\hline 3 & $\mathrm{~A}$ & 75 & CPT & 942,9 & 106 & 1048,9 \\
\hline 4 & $\mathrm{~A}$ & 100 & $\mathrm{CAB}$ & 939 & 144 & 1083 \\
\hline 5 & $\mathrm{~B}$ & 120 & $\mathrm{CAB}$ & 0 & 72 & 86,4 \\
\hline 6 & $\mathrm{~B}$ & 100 & CPT & 777 & 212 & 1186,8 \\
\hline 7 & $\mathrm{~B}$ & 105 & OPT & 0 & 148 & 177,6 \\
\hline 8 & A & 80 & $\mathrm{CCB}$ & 687,65 & 72 & 759,65 \\
\hline 9 & B & 110 & $\mathrm{CCB}$ & 0 & 144 & 172,80 \\
\hline 10 & $\mathrm{C}$ & 110 & CPT & 0 & 212 & 296,8 \\
\hline 11 & $\mathrm{~A}$ & 110 & FA & 0 & 72 & 72 \\
\hline 12 & $\mathrm{C}$ & 120 & FA & 0 & 72 & 100,8 \\
\hline 13 & $\mathrm{~B}$ & 115 & FA & 0 & 72 & 86,4 \\
\hline
\end{tabular}

Table 8. Solutions obtained through method 2 (first 13 records only)

\begin{tabular}{|r|r|r|r|r|r|r|}
\hline$\#$ & Base ID & \multicolumn{1}{|l|}{ Time (sec) } & Rank & \multicolumn{1}{|c|}{ Duty Pay } & Perdiem Pay & \multicolumn{1}{c|}{ Cost } \\
\hline 1 & A & 20 & CAB & 0 & 72 & 72 \\
\hline 2 & B & 31 & CAB & 0 & 72 & 86,4 \\
\hline 3 & B & 18 & CPT & 0 & 106 & 127,2 \\
\hline 4 & C & 27 & CAB & 563,4 & 62 & 875,6 \\
\hline 5 & B & 32 & CAB & 0 & 72 & 86,4 \\
\hline 6 & C & 26 & CPT & 0 & 212 & 296,8 \\
\hline 7 & A & 25 & OPT & 0 & 144 & 144 \\
\hline 8 & B & 15 & CCB & 229,17 & 72 & 361,4 \\
\hline 9 & B & 29 & CCB & 0 & 144 & 172,8 \\
\hline 10 & C & 23 & CPT & 0 & 212 & 296,8 \\
\hline 11 & A & 27 & FA & 0 & 72 & 72 \\
\hline 12 & C & 31 & FA & 0 & 72 & 100,8 \\
\hline 13 & B & 32 & FA & 0 & 72 & 86,4 \\
\hline
\end{tabular}


Table 9. Comparison of the results

\begin{tabular}{|l|r|r|r|r|r|}
\hline & \multicolumn{2}{|c|}{ Method 1 } & \multicolumn{2}{c|}{ Method 2 } & Met1/Met2 \\
\hline & \multicolumn{1}{|c|}{ Total } & \multicolumn{1}{c|}{ Total } & \multicolumn{1}{c|}{$\%$} & $\%$ \\
\hline Solution base: & & & & & \\
\hline - From base (A) & 7 & 47 & 3 & 20,0 & $-57,1$ \\
\hline - From base B & 6 & 40 & 7 & 47,0 & 16,7 \\
\hline - From base C & 2 & 13 & 5 & 33,0 & 150,0 \\
\hline & & & & & \\
\hline Time (avg sec) & 101 & 100 & 25 & 24,8 & $-75,3$ \\
\hline - Base A (avg) & 88 & 21 & 24 & 24,0 & $-72,7$ \\
\hline - Base B (avg) & 110 & 27 & 24 & 24,0 & $-78,2$ \\
\hline - Base C (avg) & 115 & 28 & 26 & 26,0 & $-77,4$ \\
\hline & & & & & \\
\hline Total Costs: & 7039,60 & 100 & 3839,36 & 54,5 & $-45,5$ \\
\hline Costs by Base: & & & & & \\
\hline - Base A & 4845,55 & 92,4 & 288,00 & 11,2 & $-94,0$ \\
\hline - Base B & 1796,40 & 34,3 & 1275,80 & 49,8 & $-29,0$ \\
\hline - Base C & 397,60 & 7,6 & 2275,56 & 88,8 & 472,3 \\
\hline Costs by Rank & & & & & \\
\hline - CPT & 2532,5 & 36,0 & 720,8 & 18,8 & $-71,5$ \\
\hline - FO & 720,0 & 10,2 & 499,2 & 13,0 & $-30,7$ \\
\hline - SFA & 932,5 & 13,3 & 534,2 & 13,9 & $-42,7$ \\
\hline - FA & 2854,6 & 40,6 & 2085,1 & 54,3 & $-27,0$ \\
\hline
\end{tabular}

is, in average 4 times faster than the first method in finding a solution and produces solutions that represent a decrease of $45.5 \%$ on the costs.

The Solution part of table 9 shows the number of solutions found in each operational base. The Time part of the table shows how long it took to find the solutions in each base. The Total Cost shows the cost of the solutions by each of the crew ranks involved: CPT (Captains), FO (First Officers), SFA (Senior Flight Attendants) and FA (Flight Attendants). It also shows the costs by each of the operational bases.

\section{Discussion and Conclusions}

From the results obtained we can see that our MAS obtains valid solutions faster and with less costs when compared with the current method used in a real airline company. Regarding our first hypothesis we were expecting a considerable decrease in the costs of the solutions found by our MAS. From the results obtained (see Table 9) we can see that:

\section{Cost $($ SolutionMAS(3839,36))<Cost(SolutionMet1 $(7039,60))$}

It represents a decrease of $45.5 \%$ on the costs. Our hypothesis was accepted. Of course that we cannot infer that our MAS will always produce solutions that cost 45.5\% less. It is not even possible to say that, in average, this decrease is valid. For that we need to evaluate much more situations, in different times of the year (we might have seasoned behaviors) and, then, find an average value. However, taking into consideration that our method includes information that is not available in the current method of the airline (for example, hour salary, perdiem value, lodging and 
extra-crew travel), and that this information has an immense impact on the total cost, we can state that our method will never produce more expensive solutions.

From the results we can also obtain other interesting conclusions. These conclusions can be expressed by the following formalism:

\section{Time(SolutionMAS(25s))<Time(SolutionMet1(101s)) \\ 2. Cooperation(SolutionMAS(BaseB(47\%))) > \\ Cooperation(SolutionMet1(BaseB(40\%))) and \\ Cooperation(SolutionMAS(BaseC(33\%))) > \\ Cooperation(SolutionMet1(BaseC(13\%)))}

Regarding 1) our method was $75.3 \%$ faster than method one. The use of a computerized system to find and evaluate the solutions is the reason for our method to be faster than the present, almost manual, method used in the airline. Regarding 2) we can see that the cooperation between different operational bases has increased with our method, because we evaluate all the solutions found (including the ones from different operational bases where the event happened) and choose the one with less cost. In method one, they choose the first one they find. This cooperation is also possible to be inferred from the costs by base. In Table 9 is possible to see that the costs of base $\mathrm{C}$ had an increase of $472.32 \%$ while base A and base B decreased $94 \%$ and $29 \%$, respectively. This means that our method used more resources from other bases than the base where the problem happened (base A).

Regarding our second hypothesis we expected to increase the robustness of our system using heterogeneous algorithms to find solutions to the same problem, at the same time. We were not able to collect enough data to analyze the impact on robustness as the result of using different specialized agents. Preliminary results show that, most of the times, the MAS presents at least one solution even when the human operator cannot found one. Apparently this is the result of using different techniques to tackle the problem. However, the solution might have a cost that, when compared with other ways of solving the problem (for example, cancelling the flight), might be unacceptable. This tells us that our MAS need to have access to more information. For example and in the case of cancelling the flight, it would be important to have access to the cost of compensations due to passengers in these situations.

This paper has presented a distributed multi-agent system as a possible solution to solve airline operations recovery problems, including sub-organizations with specialized agents, dedicated to solve crew, aircraft and passenger recovery problems. We have detailed the architecture of our MAS regarding the sub-organization dedicated to solve crew recovery problems, including agents, services and protocols. We have introduced a multi-criteria algorithm for selecting the solution with less cost from those proposed as part of the negotiation process. A simple example was presented, following, step-by-step, our proposed method. A case study, taken from a real scenario in an airline company where we have tested our method was also presented and we discuss the results obtained. We have shown that our method produces faster and less expensive solutions when compared with the present and almost manual method, used in the airline company.

Further work is required in testing our method for large periods of time and in different times of the year (due to seasoned behaviors). We also need to test our MAS 
with all the sub-organizations working at the same time (crew, aircraft and passenger) to see the impact that might exist in the results we have presented in this paper. Finally, we would like to apply and test the integration of the EM as presented in [10].

Acknowledgements. António Castro is grateful to TAP Portugal for the support during this research and for allowing the use of real data extracted from the information system used in TAP Portugal.

\section{References}

1. Abdelgahny, A., Ekollu, G., Narisimhan, R., Abdelgahny, K.: A Proactive Crew Recovery Decision Support Tool for Commercial Airlines during Irregular Operations. Annals of Operations Research 127, 309-331 (2004)

2. Barnhart, C., Belobaba, P., Odoni, A.: Applications of Operations Research in the Air Transport Industry. Transportation Science 37, 368-391 (2003)

3. Bellifemine, F., Caire, G., Trucco, T., Rimassa, G.: JADE Programmer's Guide. JADE 3.3, TILab S.p.A (2004)

4. Bratu, S., Barnhart, C.: Flight Operations Recovery: New Approaches Considering Passenger Recovery. Journal of Scheduling 9(3), 279-298 (2006)

5. Castro, A., Oliveira, E.: A Multi-Agent System for Intelligent Monitoring of Airline Operations. In: Proceedings of the Third European Workshop on Multi-Agent Systems, Brussels, Belgium, pp. 91-102 (2005)

6. Clausen, J., Larsen, A., Larsen, J.: Disruption Management in the Airline Industry Concepts, Models and Methods. Technical Report 2005-01, Informatics and Mathematical Modeling, Technical University of Denmark, DTU (2005)

7. Kohl, N., Larsen, A., Larsen, J., Ross, A., Tiourline, S.: Airline Disruption Management Perspectives, Experiences and Outlook. Technical Report CRTR-0407, Carmen Research (2004)

8. Kohl, N., Karish, S.: Airline Crew Rostering: Problem Types, Modeling and Optimization. Annals of Operations Research 127, 223-257 (2004)

9. Lettovsky, L.: Airline Operations Recovery: An Optimization Approach, Ph.D. Thesis, Georgia Institute of Technology, Atlanta, USA (1997)

10. Malucelli, A., Castro, A., Oliveira, E.: Crew and Aircraft Recovery Through a Multi-Agent Electronic Market. In: Proceeding of IADIS International Conference e-Commerce 2006, Barcelona, Spain, pp. 51-58. IADIS Press (2006) ISBN: 972-8924-23-2

11. Martins, J., Morgado, E.: Managing Flight Operations. In: Proceedings of ATTIS 1996, London, England (1996)

12. Rosenberger, J., Johnson, E., Nemhauser, G.: Rerouting aircraft for airline recovery, Technical Report TLI-LEC 01-04, Georgia Institute of Technology (2001)

13. Rosenberger, J., Schaefer, A., Goldsmans, D., Johnson, E., Kleywegt, A., Nemhauser, G.: A Stochastic Model of Airline Operations. Transportation Science 36(4), 357-377 (2002) 